\title{
Web Support for Weight-Loss Interventions: PREDIRCAM2 Clinical Trial Baseline Characteristics and Preliminary Results
}

\author{
Valeria Alcántara-Aragón, MD, Susana Rodrigo-Cano, RD, Ascension Lupianez-Barbero, RD, \\ María José Martinez, RN, Carmen Martinez, RN, José Tapia, MSc, José Manuel Iniesta, MSc, \\ Susana Tenes, MD, PhD, Eulalia Urgell, PhD, Gemma Navarro, RD, \\ M. Elena Hernando, PhD, Juan Francisco Merino-Torres, MD, PhD, \\ Alberto de Leiva, MD, PhD, and Cintia Gonzalez, MD, PhD
}

\begin{abstract}
An ongoing clinical trial is testing the efficacy of web telematic support in a structured program for obesity treatment and diabetes prevention. Participants were recruited from two tertiary-care hospitals and randomized to receive either a telematic intervention (TI) supported by PREDIRCAM2 web platform or a non-telematic intervention (NTI). All receive 1-year follow-up. Both interventions consist of tailored dietary and exercise prescriptions, based on a Mediterranean dietary pattern and general WHO exercise recommendations for adults. At 6 months, both groups have received 7 contacts, 3 exclusively telematic for the TI group. This is a preliminary result intention-to-treat analysis. One hundred eighty-three participants were recruited, with a mean body mass index of $34.75 \pm 2.75 \mathrm{~kg} / \mathrm{m}^{2}$. General dropout rate at 6 months was $26.8 \%$. Weight changes were statistically significant at months 3 and 6 compared to baseline, $-2.915 \pm 0.24 \mathrm{~kg},-3.29 \pm 0.36 \mathrm{~kg}$, respectively $(P<0.001)$, but not statistically significant between the $3-$ and 6-month time points $-0.37 \pm 0.21 \mathrm{~kg}(P=0.24)$. Mean group differences showed that the TI group lost $1.61 \pm 1.88 \mathrm{~kg}$ more than the NTI group $(P=0.39)$. Waist, waist/hip ratio, resting heart rate, blood pressure, HbA1c, and low-density lipoprotein cholesterol also showed statistically significant changes at 6 months, with no significant differences between groups. Weight loss in the TI group shows similar results as the usual care NTI group for weight loss and control of obesity comorbidities. At completion of the clinical trial, these results will be reevaluated to assess the potential role of web support in weight-loss maintenance and its cost-effectiveness.
\end{abstract}

Keywords: Telemedicine, Obesity, Metabolic syndrome, Lifestyle modification.

\section{Background}

$\mathbf{O}$ BESITY AND ITS COMORBIDITIES are the pandemic of our age. Despite the proliferation of health promotion programs, the prevalence of obesity has not declined and is yet continuing to rise in specific populations. ${ }^{1}$ Intensified treatments have proven to be effective for weight loss and diabetes treatment and prevention. Unfortunately, they remain unavailable in most countries given their elevated cost. In the battle against obesity and its comorbidities, telemedicine 
could offer cost-effective tools to facilitate intensified treatments. In our setting, telematic interventions (TIs) offering continued support and self-monitoring for conditions such as sleep apnea, or gestational diabetes, have shown to be useful and potentially cost-effective. ${ }^{2-5}$

PREDIRCAM2 is a web platform designed for promotion of healthy lifestyles, weight loss, weight-loss maintenance, and cardiometabolic risk prevention. ${ }^{6}$ There is an ongoing clinical trial designed to assess the potential benefit of using web support in a structured treatment program for obesity and prevention of its comorbidities: Individualized telemedical assistance for lifestyle modification in the treatment of obesity and cardiometabolic risk prevention (NCT 01919372). Recruitment has been completed and all active participants have completed the 6-month follow-up.

\section{Materials and Methods}

In accordance with the Declaration of Helsinki, the participant institutions bioethics committee approved the study protocol. Participants were recruited at the Nutrition and Endocrinology Departments of two tertiary-care hospitals. They were derived by their primary care physicians or by the hospitals' occupational health departments. Eligibility criteria were as follows: adults 18 to 65 years, body-mass index between 30 and $39.9 \mathrm{~kg} / \mathrm{m}^{2}$, no severe illnesses, no pregnancy, and not receiving medications for glucose, lipid, blood pressure, or weight control. A randomization list was generated at https://www.sealedenvelope.com/ to allocate participants in two groups.

\section{Program structure}

In the first appointment, participants underwent evaluation of inclusion criteria with medical history anamnesis and provided their signed informed consent. Blood testing and anthropometry were performed in the second appointment. In the third appointment, participants were informed of their randomization to receive either a TI supported by the PREDIRCAM2 web platform or a non-telematic intervention (NTI). All participants will receive 1-year follow-up and treatment. Both interventions consist of tailored exercise and dietary prescriptions based on a Mediterranean dietary pattern and general WHO exercise recommendations for adults. TI group participants receive a heart rate monitor watch at baseline to estimate their physical activity intensity and energy expenditure (Polar RS400). Both groups have access to the same informative material and are asked to $\log$ their food intake; TI group through the web platform and NTI group on paper. At the 6-month follow-up, the NTI group had received treatment in 7 in-person contacts, while the TI group received 4 in-person contacts, 3 telematic contacts, and continued web support. Blood testing was performed again at the 6-month follow-up.

\section{Description of the web platform}

The PREDIRCAM2 web platform (http://limbhad.gbt.tfo .upm.es:8080/) was designed as an educational tool that allows healthcare providers to tailor dietary and physical activity goals, and users to assess and progressively adjust their lifestyles. ${ }^{6}$ The exercise module receives input from the Polar RS400 heart rate monitor and also through direct manual registry from the user. The dietary module feeds on a dynamic database that grows according to the users' requests and allows them to save personal ingredient combinations. The platform provides feedback when logging dietary intake and physical activity, and also through general automatized notifications. A messaging system integrated into the

Table 1. Baseline Characteristics of the Study Population

\begin{tabular}{lcccc}
\hline & General, $\mathrm{n}=183$ & TI group, $\mathrm{n}=91$ & NTI group, $\mathrm{n}=92$ & $\mathrm{P}$ \\
\hline Age (years) & $44.5 \pm 10.8$ & $43.9 \pm 11.2$ & $44.6 \pm 11.5$ & 0.6688 \\
Females (\%) & 83.6 & 86 & 82 & 0.4437 \\
High education level (\%) & 54 & 61 & 47 & 0.0446 \\
Smokers (\%) & 16 & 18 & 13 & 0.3271 \\
Ex-smokers (\%) & 28 & 27 & 30 & 0.7081 \\
Hypertension (\%) & 5 & 5.4 & 5.5 & 0.9859 \\
Type 2 diabetes mellitus (\%) & 2 & 4.4 & 0 & 0.0449 \\
Dyslipidemia (\%) & 11 & 11 & 12 & 0.8386 \\
BMI (kg/m ${ }^{2}$ ) & $34.75 \pm 2.75$ & $34.5 \pm 2.7$ & $35 \pm 2.8$ & 0.2293 \\
Weight (kg) & $94.17 \pm 12.68$ & $93.2 \pm 12.1$ & $95.1 \pm 13$ & 0.2987 \\
Waist (cm) & $105.93 \pm 9.5$ & $104.71 \pm 9$ & $105.87 \pm 10.1$ & 0.4129 \\
WHR & $0.912 \pm 0.08$ & $0.911 \pm 0.08$ & $0.913 \pm 0.09$ & 0.8737 \\
RHR (bpm) & $73.2 \pm 12.1$ & $74.1 \pm 11.4$ & $74.2 \pm 11.8$ & 0.9149 \\
SBP (mmHg) & $128.85 \pm 14.6$ & $129.4 \pm 14.4$ & $127.5 \pm 14.4$ & 0.3647 \\
DBP (mmHg) & $83.81 \pm 9.18$ & $84.3 \pm 8.9$ & $82.4 \pm 8.4$ & 0.1386 \\
HbA1c (\%) & $5.47 \pm 0.36$ & $5.44 \pm 0.40$ & $5.41 \pm 0.33$ & 0.5149 \\
Insulin (pmol/L) & $88.42 \pm 42.75$ & $94.33 \pm 47.74$ & $92.26 \pm 49.94$ & 0.7796 \\
LDL cholesterol (mg/dL) & $120.4 \pm 29$ & $120.8 \pm 26.4$ & $123.7 \pm 31.5$ & 0.5031 \\
Non-HDL cholesterol (mg/dL) & $138.98 \pm 32$ & $140.97 \pm 31.02$ & $142.98 \pm 33.6$ & 0.6764 \\
HDL (mg/dL) & $55.24 \pm 14$ & $54.4 \pm 13$ & $53.4 \pm 14$ & 0.6275 \\
LDL/ApoB & $1.21 \pm 0.21$ & $1.22 \pm 0.2$ & $1.21 \pm 0.2$ & 0.7594 \\
Triglycerides (mg/dL) & $93.71 \pm 36$ & $104.03 \pm 61.50$ & $99.41 \pm 46.33$ & 0.5683 \\
HOMA-IR & $2.93 \pm 1.57$ & $3.09 \pm 1.68$ & $3.04 \pm 1.74$ & 0.8673 \\
\hline
\end{tabular}

BMI, body mass index; DBP, diastolic blood pressure; HDL, high-density lipoprotein; HOMA-IR; LDL, low-density lipoprotein; NTI, non-telematic intervention; RHR, resting heart rate; SBP, systolic blood pressure; TI, telematic intervention; WHR, waist/hip ratio. 
platform enables the users to communicate with healthcare providers. Also, the platform includes a link to a private group on Facebook ${ }^{\mathbb{B}}$, where users interact among themselves and with healthcare providers.

\section{Statistical analysis}

Sample size was determined to be 111 participants per group, with an $\alpha$ unilateral error of $5 \%$ and power of $80 \%$, for a tolerated difference of $2 \mathrm{~kg}$ and standard deviation of $\pm 6 \mathrm{~kg}$. The dropout of a third participating center limited our total sample size to 183 participants. Normal distribution was assessed by box-plot evaluation. Two-way ANOVAs were performed to assess the effect of group and time on our study variables: weight, waist, waist/hip ratio (WHR), HbA1c, resting heart rate (RHR), systolic blood pressure (SBP), diastolic blood pressure (DBP), insulin, homeostatic model assessment for insulin resistance (HOMA-IR), and blood lipids. There was homogeneity of variances, as assessed by Levene's test of homogeneity. The Greenhouse-Geisser correction was applied whenever we encountered sphericity violations for the two-way interactions. To deal with missing data, three types of analyses were performed: completers only per protocol (PP) analysis and two intention-to-treat (ITT) analyses. The strategies for ITT analyses were lastobservation-carried-forward (LOCF) and multiple imputation (MI). Values of $P<0.05$ were considered significant. Imputation process and statistical analysis were performed using SPSS package (version 23.0 for Mac OS; SPSS, Inc., Chicago, IL).

\section{Results}

Table 1 shows the baseline characteristics of the study population $(n=183)$. There were no significant differences between groups at baseline, except for education level and type 2 diabetes mellitus (T2DM) diagnosis. A larger percentage of participants in the TI group had a high education level, $61.5 \%$ having at least one higher education degree compared with $46.7 \%$ of the NTI group $(P=0.0446)$. All participants diagnosed with diabetes were in the TI group ( $4.4 \%$ vs. $0 \%, P=0.0449$ ). Their primary care physicians had diagnosed them before recruitment, following the American Diabetes Association's diagnosis criteria. The general dropout rate was $11.5 \%$ at 3 months and increased to $26.8 \%$ at 6 months. Dropout differential rates at 6 months were $34 \%$ for the TI group and $19.6 \%$ for the NTI group. The most frequently reported reason for dropout for both groups was finding the intervention too timeconsuming, followed by a self-reported lack of motivation, and family issues. For detailed information on participant flow in the study (Supplementary Fig. S1; Supplementary Data are available at http://online.liebertpub.com/suppl/doi/ 10.1089/dia.2017.0456). Figure 1 shows weight by group from three different types of analyses. Table 2 shows the effect of time on study variables.

\section{Completers only: PP analysis}

Weight changes were statistically significant at 3 and 6 months compared to baseline, $-4.0 \pm 0.27 \mathrm{~kg}$ and $-4.5 \pm 0.47 \mathrm{~kg}$, respectively $(P<0.001)$; but not statistically significant between

\section{善部}
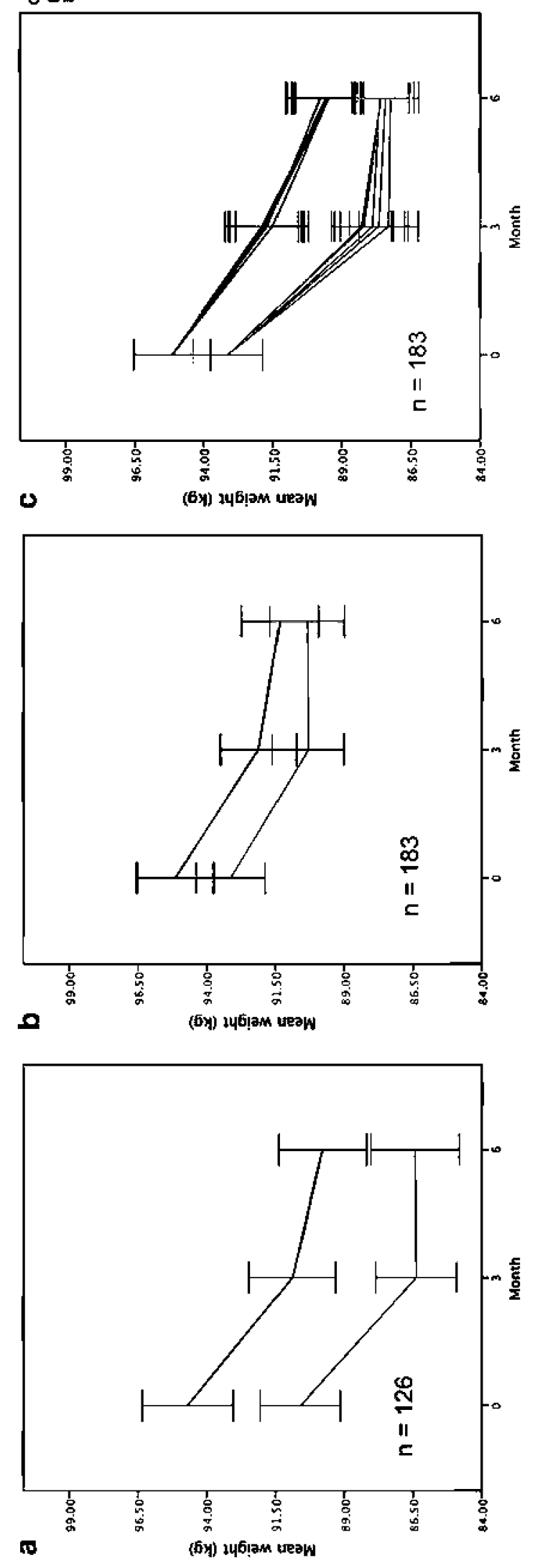


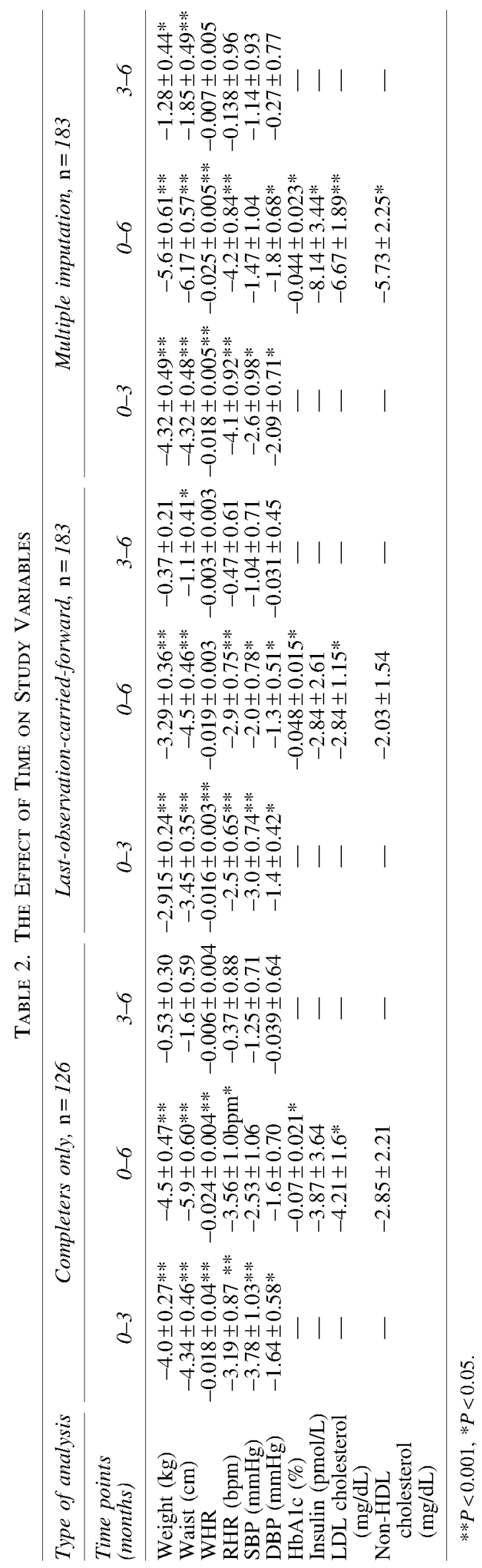

the 3 - and 6-month time points $-0.53 \pm 0.30 \mathrm{~kg}(P=0.24)$. Mean group differences showed the TI group $(n=55)$ lost a mean of $-3.99 \pm 2.23 \mathrm{~kg}$ more than the NTI group $(n=71)$; this difference, however, was not statistically significant $(P=0.075)$. Mean differences between groups were not statistically significant for waist, WHR, HbA1c, RHR, SBP, DBP, HbA1c, HOMA-IR, and blood lipids, and no significant changes were detected on high-density lipoprotein (HDL), non-HDL cholesterol, low-density lipoprotein (LDL)/ApoB index, triglycerides, insulin, or HOMA-IR at 6 months (data not shown).

\section{ITT analysis: $L O C F$}

ITT analysis with LOCF showed weight changes were statistically significant at 3 and 6 months compared to baseline, $-2.915 \pm 0.24 \mathrm{~kg}$ and $-3.29 \pm 0.36 \mathrm{~kg}$, respectively $(P<0.001)$, but not statistically significant between the 3- and 6-month time points $-0.37 \pm 0.21 \mathrm{~kg}(P=0.24)$. Mean group differences showed the TI group $(n=91)$ lost $1.61 \pm 1.88 \mathrm{~kg}$ more than the NTI group $(n=92)$; this difference, however, was not statistically significant $(P=0.39)$. ITT analysis with LOCF showed no significant differences between groups for waist, WHR, HbA1c, RHR, SBP, DBP, HbA1c, HOMA-IR, and blood lipids. No significant changes were detected on HDL, nonHDL cholesterol, LDL/ApoB index, triglycerides, insulin, or HOMA-IR at 6 months (data not shown).

\section{ITT analysis: $M I$}

ITT analysis with MI showed weight changes were statistically significant at all time points. Between 3 and 6 months compared to baseline, $-4.32 \pm 0.49 \mathrm{~kg}$ and $-5.6 \pm 0.61 \mathrm{~kg}$, respectively $(P<0.001)$; and between the 3 - and 6-month time points: $-1.28 \pm 0.44 \mathrm{~kg}(P=0.011)$. Mean group differences showed the TI group $(n=91)$ lost $-2.66 \pm 1.61 \mathrm{~kg}$ more than the NTI group $(n=92)$, however, this difference was not statistically significant $(P=0.101)$. ITT analysis with $\mathrm{MI}$ showed no significant differences between groups for waist, WHR, HbA1c, RHR, SBP, DBP, or blood lipids. HDL, triglycerides, LDL/ApoB index, or HOMA-IR did not show any significant changes at 6 months (data not shown).

\section{Conclusions}

Applied health technologies in lifestyle interventions for obesity have been tried in a wide variety of combinations to provide telematic support for weight loss in countries from North America, Australia, and Northern Europe. ${ }^{7-11}$ To our knowledge, this is the first clinical trial of its kind to be held in a Mediterranean clinical setting.

The magnitude of weight changes at 6 months was similar to reports from other lifestyle interventions and to other technology-based weight-loss intervention programs. ${ }^{12-14}$ Blood lipids and $\mathrm{HbAlc}$ changes were mild, however, this can be expected from a mostly nondiabetic population whose blood glucose and lipids were controlled without requiring any medications at recruitment.

Individualization, self-tracking, and e-coaching are key characteristics that have been related to improved health outcomes, usability, and adherence to e-health interventions. ${ }^{11}$ The PREDIRCAM2 web platform integrates these key characteristics into a comprehensive lifestyle intervention. Weight, 
waist, WHR, HbAlc, and lipid changes were not significantly different between groups, suggesting that a substitution of $43 \%$ of the contacts with telematic support through the PREDRICAM2 web platform could be feasible. Continued web support could allow for both more contacts and an individualized approach.

The main limitation of this study is that it is underpowered to show significant differences between groups. High dropout rates are common in obesity intervention trials, limiting results and demanding for strategies to deal with missing data. Intensified intervention programs for obesity usually require frequent contacts with healthcare providers for reinforcement. Previous studies suggest that face-to-face contacts are required to provide clear instructions for implementation and engage patients in their therapy; therefore, not all the contacts can be substituted by any telematic strategy. ${ }^{11}$ The amount of face-to-face contacts required by each individual may vary. ${ }^{15}$ This may be a reason for the higher dropout rate seen in the TI group, since the use of technology in itself was not a frequently reported reason for dropout. Despite these limitations, the same trends were encountered for weight, waist, and WHR across all the analysis approaches.

Across the different strategies for dealing with missing data, the TI group exhibits the same weight-loss trend with an initial steep slope before the 3-month time point that tends to stabilize toward 6 months. Waist and WHR show similar trends. This trend may reflect the participants' motivation and adherence to treatment. There is a possible motivational effect in the use of technology that tends to wear off at 2 or 3 months, with consequent drops in adherence. The use of technologies in lifestyle interventions, such as webs, mobile applications, or physical activity trackers, has shown similar trends, with adherence rates tending to decline at 2 to 3 months of follow-up. $9,16,17$

The TI group shows similar preliminary results for weight loss and control of obesity comorbidities when compared to usual care. At the completion of the ongoing clinical trial, these results will be reevaluated to assess the potential role of web support for weight-loss maintenance as well as its costeffectiveness.

\section{Acknowledgment}

Assistance provided by Dr. Ignasi Gich from the Statistics and Epidemiology Department of Santa Creu I Sant Pau Hospital was greatly appreciated in the preparation of this article. The authors appreciate the support and collaboration provided to this project by Dr. José Maria Cubero, Eulalia Brugués and the Endocrinology nurse team at Santa Creu I Sant Pau Hospital.

\section{Author Disclosure Statement}

The web platform PREDIRCAM2 was designed in a project funded by Instituto Carlos Tercero de Madrid (FIS PI12/00931) at the Endocrinology and Nutrition Department of Santa Creu I Sant Pau Hospital in Barcelona, and Centro de Tecnología Biomédica, ETSI de Telecommunicación at Universidad Politécnica de Madrid, Spain. V.A.-A. and A.L. have received scholarships from the Catalan administration (Departament d'Economía I Coneixement de la Generalitat de Catalunya, Fondo So- cial Europeo, AGAUR-FI-DGR). V.A.-A. received an award from Sociedad Española de Endocrinología y $\mathrm{Nu}-$ trición in alliance with MSD and the project received media resource collaboration from Roche Diabetes Care.

\section{References}

1. OECD: Obesity Update 2017 (OECD). www.oecd.org/els/ health-systems/Obesity-Update-2017.pdf (accessed November 27, 2017).

2. Caballero-Ruiz E, García-Sáez G, Rigla M, et al.: A webbased clinical decision support system for gestational diabetes: automatic diet prescription and detection of insulin needs. Int J Med Inform 2017;102:35-49.

3. Galiano-Castillo N, Cantarero-Villanueva I, Fernández-Lao C, et al.: Telehealth system: a randomized controlled trial evaluating the impact of an internet-based exercise intervention on quality of life, pain, muscle strength, and fatigue in breast cancer survivors: telehealth system in breast cancer. Cancer 2016;122:3166-3174.

4. Isetta V, Torres M, González K, et al.: A New mHealth application to support treatment of sleep apnoea patients. J Telemed Telecare 2017;23:14-18.

5. Fuertes-Guiró F, Vitali-Erion E, Rodriguez-Franco A: A program of telementoring in laparoscopic bariatric surgery. Minim Invasive Ther Allied Technol 2016;25:8-14.

6. González C, Herrero P, Cubero JM, et al.: PREDIRCAM eHealth platform for individualized telemedical assistance for lifestyle modification in the treatment of obesity, diabetes, and cardiometabolic risk prevention: a pilot study. J Diabetes Sci Technol 2013;7:888-897.

7. Hutchesson MJ, Rollo ME, Krukowski R, et al.: eHealth interventions for the prevention and treatment of overweight and obesity in adults: a systematic review with meta-analysis: eHealth interventions for obesity in adults. Obes Rev 2015;16:376-392.

8. Coons MJ, DeMott A, Buscemi J, et al.: Technology interventions to curb obesity: a systematic review of the current literature. Curr Cardiovasc Risk Rep 2012;6:120-134.

9. Lyons EJ, Lewis ZH, Mayrsohn BG, et al.: Behavior change techniques implemented in electronic lifestyle activity monitors: a systematic content analysis. J Med Internet Res 2014;16:e192.

10. Sorgente A, Pietrabissa G, Manzoni GM, et al.: Web-based interventions for weight loss or weight loss maintenance in overweight and obese people: a systematic review of systematic reviews. J Med Internet Res 2017;19:e229.

11. Lentferink AJ, Oldenhuis HK, de Groot M, et al.: Key components in eHealth interventions combining selftracking and persuasive eCoaching to promote a healthier lifestyle: a scoping review. J Med Internet Res 2017;19: e277.

12. De Luis DA, Izaola O, Primo D, et al.: Biochemical, anthropometric and lifestyle factors related with weight maintenance after weight loss secondary to a hypocaloric mediterranean diet. Ann Nutr Metab 2017;217-223.

13. Gepner Y, Shelef I, Schwarzfuchs D, et al.: Effect of distinct lifestyle interventions on mobilization of fat storage pools: the central MRI randomized controlled trial. Circulation 2017; pii: CIRCULATIONAHA.117.030501.

14. Haggerty AF, Hagemann A, Barnett M, et al.: A randomized, controlled, multicenter study of technology-based weight loss interventions among endometrial cancer survivors: technology-based weight loss and endometrial cancer. Obesity 2017;25:S102-S108. 
15. Rafiei N, Gill T: Identification of factors contributing to successful self-directed weight loss: a qualitative study. J Hum Nutr Diet 2017. DOI:10.1111/jhn.12522.

16. Hermsen S, Moons J, Kerkhof P, et al.: Determinants for sustained use of an activity tracker: observational study. JMIR MHealth UHealth 2017;5:e164.

17. Cadmus-Bertram L, Marcus BH, Patterson RE, et al.: Use of the fitbit to measure adherence to a physical activity intervention among overweight or obese, postmenopausal women: self-monitoring trajectory during 16 weeks. JMIR MHealth UHealth 2015;3:e96. 


\section{Supplementary Data}

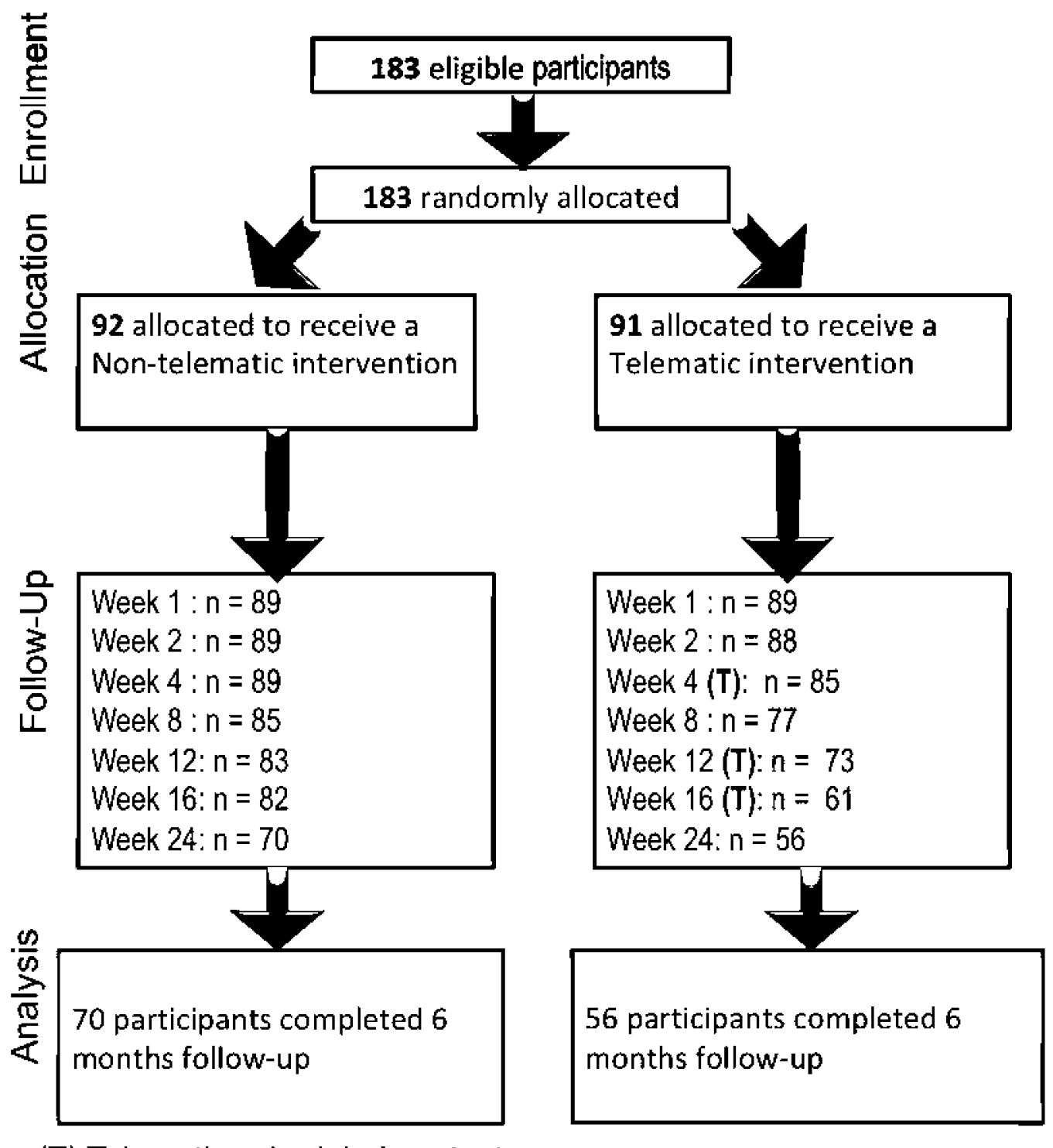

(T) Telematic scheduled contacts

SUPPLEMENTARY FIG. S1. Flow of participants in the first 6 months of PREDIRCAM2 clinical trial. Telematic scheduled contacts are marked as (T). 